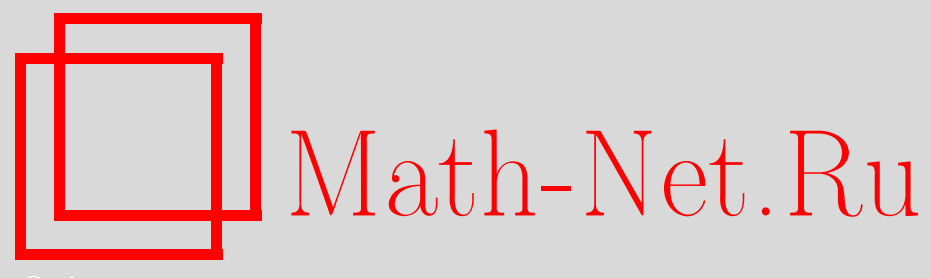

С. Де Никола, Р. Феделе, М. А. Манько, В. И. Манько, Новые неравенства для томограмм в вероятностном представлении квантовых состояний, ТМФ, 2007, том 152, номер 2, 241-247

DOI: https://doi.org/10.4213/tmf6084

Использование Общероссийского математического портала Math-Net.Ru подразумевает, что вы прочитали и согласны с пользовательским соглашением http://www . mathnet.ru/rus/agreement

Параметры загрузки:

IP : 54.157 .27 .8

26 апреля 2023 г., $17: 33: 50$

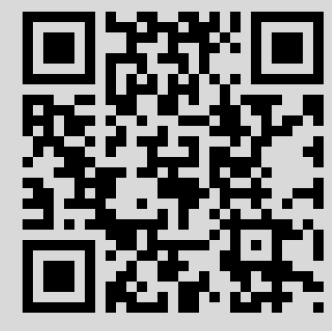




\title{
НОВЫЕ НЕРАВЕНСТВА ДЛЯ ТОМОГРАММ В ВЕРОЯТНОСТНОМ ПРЕДСТАВЛЕНИИ КВАНТОВЫХ СОСТОЯНИЙ
}

\begin{abstract}
В рамках вероятностного представления квантовой механики обсуждаются новые неравенства для симплектических томограмм квантовых состояний и связь этих неравенств с энтропийными соотношениями неопределенностей.
\end{abstract}

Ключевые слова: томографическое отображение, симплектическая томография, энтропийные соотношения неопределенностей, неравенства для томограмм.

\section{1. ВВЕДЕНИЕ}

Существуют несколько эквивалентных формулировок квантовой механики (см., например, обзор [1]). Недавно была введена новая формулировка квантовой механики, названная вероятностным представлением квантовой механики [2], [3]. В рамках этой формулировки квантовые состояния описываются стандартными распределениями вероятностей вместо волновых функций или матриц плотности. Вероятностное представление квантовой механики эквивалентно всем другим представлениям, но оно оказывается более удобным при рассмотрении некоторого класса квантовых проблем, для которых может быть использован хорошо разработанный математический аппарат теории вероятности.

Энтропия Шеннона [4] является функционалом, характеризующим любое распределение вероятности; она была использована для того, чтобы ввести томографическую энтропию для непрерывных переменных [5] и аналитических сигналов [6],

*Istituto di Cibernetica "Eduardo Caianiello" del CNR Comprensorio "A. Olivetti" Fabbr. 70, Via Campi Flegrei 34, I-80078 Pozzuoli (NA), Italy. E-mail: s.denicola@cib.na.cnr.it

${ }^{\dagger}$ Dipartimento di Scienze Fisiche, Università "Federico II" di Napoli and Istituto Nazionale di Fisica Nucleare, Sezione di Napoli, Complesso Universitario di M. S. Angelo, Via Cintia, I-80126 Napoli, Italy. E-mail: renato.fedele@na.infn.it

‡Физический институт им. П. Н. Лебедева РАН, Москва, Россия. E-mail: mmanko@sci.lebedev.ru,manko@sci.lebedev.ru 
а также для спиновых томографических вероятностей [7]. Было показано, что вероятностное представление, рассмотренное в работах [8]-[10], реализует новую версию процедуры квантования, основанную на формализме звездочного произведения. Метод звездочного произведения и различные свойства томографических энтропий в применении к квантовой информации изучались в [11], где энтропия Реньи [12] рассматривалась с использованием спиново-томографических вероятностей (томограмм).

Существуют неравенства для энтропии Шеннона, связанные с распределениями вероятностей координаты и импульса (см., например, обзор [13]). Недавно были найдены новые энтропийные соотношения неопределенностей [14], связанные с энтропией Реньи [12]. С помощью энтропийных соотношений неопределенностей в [15] получены новые неравенства для томографических распределений вероятностей для непрерывных переменных (названных симплектическими томограммами).

Целью настоящей работы является обзор новых энтропийных соотношений неопределенностей и получение новых неравенств для томограмм квантовых состояний в многомодовом случае.

Статья построена следующим образом. В разделе 2 мы обсудим известные энтропийные соотношения неопределенностей для одномодового и многомодового случаев. В разделе 3 мы представим обзор свойств томографических энтропий, следуя работам [5]-[7]. В разделе 4 мы рассмотрим интегральные неравенства для симплектических томограмм в одномодовом случае. В разделе 5 мы изучим интегральные неравенства для оптических и симплектических томограмм в многомодовом случае. Итоги подведем в разделе 6.

\section{2. ЭНТРОПИЙНЫЕ СООТНОШЕНИЯ НЕОПРЕДЕЛЕННОСТЕЙ}

Если квантовое состояние частицы описывается волновой функцией $\psi(x)$ в координатном представлении (или волновой функцией $\widetilde{\psi}(p)$ в импульсном представлении), то энтропии Шеннона $S_{x}$ и $S_{p}$, связанные с двумя распределениями вероятностей $|\psi(x)|^{2}$ и $|\widetilde{\psi}(p)|^{2}$, задаются следующими интегралами:

$$
\begin{aligned}
S_{x} & =-\int|\psi(x)|^{2} \ln |\psi(x)|^{2} d x, \\
S_{p} & =-\int|\widetilde{\psi}(p)|^{2} \ln |\widetilde{\psi}(p)|^{2} d p .
\end{aligned}
$$

Эти энтропии удовлетворяют энтропийному соотношению неопределенностей [13], [16], [17]

$$
S_{x}+S_{p} \geqslant \ln (\pi e) .
$$

Для матриц плотности $\rho(x, y)$ и $\rho\left(p_{x}, p_{y}\right)$ квантового состояния, описываемого соответственно в координатном и импульсном представлениях, энтропии Шеннона определяются как

$$
\begin{aligned}
& S_{x}=-\int \rho(x, x) \ln \rho(x, x) d x \\
& S_{p}=-\int \rho(p, p) \ln \rho(p, p) d p .
\end{aligned}
$$


Эти энтропии удовлетворяют тому же неравенству (2).

В многомодовом случае энтропии

$$
\begin{aligned}
& S_{\vec{x}}=-\int \rho(\vec{x}, \vec{x}) \ln \rho(\vec{x}, \vec{x}) d \vec{x}, \\
& S_{\vec{p}}=-\int \rho(\vec{p}, \vec{p}) \ln \rho(\vec{p}, \vec{p}) d \vec{p}
\end{aligned}
$$

удовлетворяют энтропийному соотношению неопределенностей с дополнительным множителем:

$$
S_{\vec{x}}+S_{\vec{p}} \geqslant N \ln (\pi e)
$$

причем $N$ является числом степеней свободы.

Энтропийные соотношения неопределенностей (2) и (5) могут быть интерпретированы как ограничения, накладываемые на матрицы плотности. Эти ограничения связаны с условиями положительности оператора плотности любого квантового состояния.

\section{3. ТОМОГРАММЫ И ТОМОГРАФИЧЕСКИЕ ЭНТРОПИИ}

В работе [2] была предложена новая формулировка квантовой механики. В рамках этой формулировки квантовое состояние, описываемое томографическим распределением вероятности $w(X, \mu, \nu)$ (названным симплектической томограммой), связано с оператором плотности $\hat{\rho}$ следующей формулой [18]:

$$
w(X, \mu, \nu)=\operatorname{Tr}(\hat{\rho} \delta(X-\mu \hat{q}-\nu \hat{p})) .
$$

Обратное преобразование имеет вид [19]

$$
\hat{\rho}=\frac{1}{2 \pi} \int w(X, \mu, \nu) e^{i(X-\mu \hat{q}-\nu \hat{p})} d X d \mu d \nu
$$

где $\hat{q}$ и $\hat{p}$ являются соответственно операторами координаты и импульса, а $X, \mu$ и $\nu$ - действительные переменные. Переменная $X$ - это случайная координата, которая измеряется в некоторой системе отсчета в фазовом пространстве, определяемой двумя действительными параметрами $\mu=s \cos \theta$ и $\nu=s^{-1} \sin \theta$. Угол $\theta$ является углом поворота осей в фазовом пространстве, а параметр сжатия $s$ определяет новый масштаб в этой системе координат. Таким образом, имеют место условие неотрицательности

$$
w(X, \mu, \nu) \geqslant 0
$$

и условие нормировки плотности томографической вероятности

$$
\int w(X, \mu, \nu) d X=1 .
$$

Если $s=1$, то томограмма называется оптической;

$$
w(X, \theta)=\operatorname{Tr}(\hat{\rho} \delta(X-\hat{q} \cos \theta-\hat{p} \sin \theta)),
$$


причем

$$
w(X, \mu=\cos \theta, \nu=\sin \theta)=w(X, \theta) .
$$

Такая томограмма использовалась для измерения квантовых состояний фотонов [20]. Симплектическая томограмма удовлетворяет условию однородности

$$
w(\lambda X, \lambda \mu, \lambda \nu)=\frac{1}{|\lambda|} w(X, \mu, \nu) .
$$

Вследствие того, что оптическая томограмма $w(X, \theta)$ и симплектическая томограмма $w(X, \mu, \nu)$ являются стандартными плотностями вероятностей, мы использовали в работах [5], [6] определение Шеннона для введения томографических энтропий

$$
\begin{aligned}
S(\mu, \nu) & =-\int w(X, \mu, \nu) \ln w(X, \mu, \nu) d X, \\
S(\theta) & =-\int w(X, \theta) \ln w(X, \theta) d X .
\end{aligned}
$$

Энтропия фон Неймана квантового состояния

$$
S_{\mathrm{N}}=-\operatorname{Tr}(\hat{\rho} \ln \hat{\rho})
$$

равна нулю для всех чистых квантовых состояний.

Томографические энтропии $S(\theta)$ и $S(\mu, \nu)$ различны для разных чистых состояний.

Из свойства однородности томограммы (12) вытекает следующее свойство томографической энтропии [21]:

$$
S\left(\sqrt{\mu^{2}+\nu^{2}} \cos \theta, \sqrt{\mu^{2}+\nu^{2}} \sin \theta\right)-\frac{1}{2} \ln \left(\mu^{2}+\nu^{2}\right)=f(\theta),
$$

откуда следует, что томографическая энтропия фактически зависит только от угловой переменной.

\section{4. ТОМОГРАФИЧЕСКОЕ ЭНТРОПИЙНОЕ СООТНОШЕНИЕ НЕОПРЕДЕЛЕННОСТИ ДЛЯ ОДНОЙ СТЕПЕНИ СВОБОДЫ}

Недавно были получены новые неравенства для томографических энтропий и томограмм квантовых состояний для непрерывных переменных [15], [21]. Представим их здесь для одномерного случая. Как было показано в [6], томограмма квантового состояния (6) может рассматриваться как распределение вероятности координаты для состояния некоторого "искусственного квантового гармонического осциллятора", эволюционирующего из начального состояния $\hat{\rho}(0)$ в состояние $\hat{\rho}(t)$. В силу этого факта периодическое во времени движение осциллятора обеспечивает превращение плотности вероятности координаты в плотность вероятности импульса после прохождения одной четверти периода колебаний. Поэтому энтропии и неравенство (2) для них могут быть рассчитаны для томограмм любого квантового состояния; в результате получаем следующее неравенство:

$$
S(\theta)+S(\theta+\pi / 2) \geqslant \ln (\pi e) .
$$


Это неравенство дает интегральное условие для оптической томограммы квантового состояния:

$$
\int[w(X, \theta) \ln w(X, \theta)+w(X, \theta+\pi / 2) \ln w(X, \theta+\pi / 2)] d X+\ln (\pi e) \leqslant 0 .
$$

Оптическая томограмма, измеряемая в экспериментах с фотонами [20], и новое неравенство (17) могут быть использованы для дополнительной проверки полученных экспериментальных результатов.

\section{5. НЕРАВЕНСТВА С ДОПОЛНИТЕЛЬНЫМИ ПАРАМЕТРАМИ ДЛЯ ТОМОГРАММ}

В работе [14] было получено новое соотношение неопределенностей для энтропии Реньи, связывающее распределения вероятностей координаты и импульса квантового состояния с оператором плотности $\hat{\rho}$. Это соотношение имеет вид

$$
\begin{gathered}
\frac{1}{1-\alpha} \ln \left\{\int_{-\infty}^{\infty}[\rho(p, p)]^{\alpha} d p\right\}+\frac{1}{1-\beta} \ln \left\{\int_{-\infty}^{\infty}[\rho(x, x)]^{\beta} d x\right\} \geqslant \\
\geqslant-\frac{1}{2(1-\alpha)} \ln \frac{\alpha}{\pi}-\frac{1}{2(1-\beta)} \ln \frac{\beta}{\pi},
\end{gathered}
$$

причем на положительные параметры $\alpha$ и $\beta$ наложено условие

$$
\frac{1}{\alpha}+\frac{1}{\beta}=2
$$

Используя тот же аргумент, что привлекался при выводе неравенства (18), получаем следующее условие на оптическую томограмму [21]:

$$
\begin{aligned}
\frac{q-1}{q} \ln \{ & \left.\int_{-\infty}^{\infty}\left[w\left(X, \theta+\frac{\pi}{2}\right)\right]^{1 /(1-q)} d X\right\}+ \\
& +\frac{q+1}{q} \ln \left\{\int_{-\infty}^{\infty}[w(X, \theta)]^{1 /(1+q)} d X\right\} \geqslant \\
\geqslant & \frac{1}{2}\left\{\frac{q-1}{q} \ln [\pi(1-q)]+\frac{q+1}{q} \ln [\pi(1+q)]\right\},
\end{aligned}
$$

причем параметр $q$ определяется из соотношения $\alpha=(1-q)^{-1}$.

Выпишем далее новое неравенство для симплектической томограммы в одномерном случае. Оно имеет вид

$$
\begin{aligned}
& \frac{q-1}{q} \ln \left\{\int_{-\infty}^{\infty}\left[w\left(X,-\sqrt{\mu^{2}+\nu^{2}} \sin \theta, \sqrt{\mu^{2}+\nu^{2}} \cos \theta\right)\right]^{1 /(1-q)} d X\right\}+ \\
& \quad+\frac{q+1}{q} \ln \left\{\int_{-\infty}^{\infty}\left[w\left(X, \sqrt{\mu^{2}+\nu^{2}} \cos \theta, \sqrt{\mu^{2}+\nu^{2}} \sin \theta\right)\right]^{1 /(1+q)} d X\right\} \geqslant \\
& \geqslant \frac{1}{2}\left\{\frac{q-1}{q} \ln [\pi(1-q)]+\frac{q+1}{q} \ln [\pi(1+q)]\right\} .
\end{aligned}
$$


Это неравенство можно интерпретировать как обобщение неравенства (18), обеспечивающее переход от оптической к симплектической томограмме.

Если использовать неравенство для энтропии Реньи, заимствованное из [14], то приведенное выше условие для томограммы квантового состояния может быть также обобщено на многомерный случай. Тогда для симплектической томограммы квантового состояния с оператором плотности $\hat{\rho}$, определенной как

$$
\begin{aligned}
& w\left(X_{1}, X_{2}, \ldots, X_{N}, \mu_{1}, \mu_{2}, \ldots, \mu_{N}, \nu_{1}, \nu_{2}, \ldots, \nu_{N}\right)= \\
& \quad=\operatorname{Tr}\left[\hat{\rho} \delta\left(X_{1}-\mu_{1} \hat{q}_{1}-\nu_{1} \hat{p}_{1}\right) \delta\left(X_{2}-\mu_{2} \hat{q}_{2}-\nu_{2} \hat{p}_{2}\right) \ldots \delta\left(X_{N}-\mu_{N} \hat{q}_{N}-\nu_{N} \hat{p}_{N}\right)\right],
\end{aligned}
$$

где $\hat{q}_{k}$ и $\hat{p}_{k}(k=1,2, \ldots, N)$ соответственно операторы координат и импульсов, получаем

$$
\begin{aligned}
& \frac{q-1}{q} \ln \left\{\int _ { - \infty } ^ { \infty } \left[w \left(X_{1}, X_{2}, \ldots, X_{N},-\sqrt{\mu_{1}^{2}+\nu_{1}^{2}} \sin \theta_{1}, \ldots\right.\right.\right. \\
& \left.\left.\left.\ldots,-\sqrt{\mu_{N}^{2}+\nu_{N}^{2}} \sin \theta_{N}, \sqrt{\mu_{1}^{2}+\nu_{1}^{2}} \cos \theta_{1}, \ldots, \sqrt{\mu_{N}^{2}+\nu_{N}^{2}} \cos \theta_{N}\right)\right]^{1 /(1-q)} d \vec{X}\right\}+ \\
& \quad+\frac{q+1}{q} \ln \left\{\int _ { - \infty } ^ { \infty } \left[w \left(X_{1}, X_{2}, \ldots, X_{N}, \sqrt{\mu_{1}^{2}+\nu_{1}^{2}} \cos \theta_{1}, \ldots\right.\right.\right. \\
& \left.\left.\left.\quad \ldots, \sqrt{\mu_{N}^{2}+\nu_{N}^{2}} \cos \theta_{N}, \sqrt{\mu_{1}^{2}+\nu_{1}^{2}} \sin \theta_{1}, \ldots, \sqrt{\mu_{N}^{2}+\nu_{N}^{2}} \sin \theta_{N}\right)\right]^{1 /(1+q)} d \vec{X}\right\} \geqslant \\
& \geqslant
\end{aligned}
$$

Для оптической томограммы $w\left(X_{1}, \ldots, X_{N}, \theta_{1}, \ldots, \theta_{N}\right)$ неравенство (24) принимает вид

$$
\begin{aligned}
\frac{q-1}{q} \ln \{ & \left.\int_{-\infty}^{\infty}\left[w\left(X_{1}, X_{2}, \ldots, X_{N}, \theta_{1}, \ldots, \theta_{N}\right)\right]^{1 /(1-q)} d \vec{X}\right\}+ \\
& +\frac{q+1}{q} \ln \left\{\int_{-\infty}^{\infty}\left[w\left(X_{1}, X_{2}, \ldots, X_{N}, \theta_{1}+\pi / 2, \ldots, \theta_{N}+\pi / 2\right)\right]^{1 /(1+q)} d \vec{X}\right\} \geqslant \\
\geqslant & \frac{N}{2}\left\{\frac{q-1}{q} \ln [\pi(1-q)]+\frac{q+1}{q} \ln [\pi(1+q)]\right\} .
\end{aligned}
$$

Для гауссовых томограмм неравенства (24) и (25) превращаются в равенства. В пределе $q \rightarrow 0$ они переходят в энтропийные соотношения неопределенностей, полученные в [15], [21].

\section{6. ЗАКЛЮЧЕНИЕ}

Суммируем основные результаты работы. В рамках вероятностного представления квантовой механики дан обзор новых соотношений неопределенностей. Эти энтропийные соотношения неопределенностей представлены в виде интегральных условий для томограмм квантовых состояний (заметим, что томограммы содержат полную информацию об этих состояниях). На основе недавно обнаруженного соотношения неопределенности для энтропии Реньи квантовых состояний [14] 
получены новые неравенства, содержащие дополнительный параметр, для некоторых интегральных выражений для томограмм квантовых состояний. Особый интерес эти условия представляют для одномодовых и многомодовых оптических томограмм вследствие того, что эти томограммы можно измерить непосредственно в квантово-оптических экспериментах [20]. Мы надеемся вывести аналогичные новые неравенства для томограмм, зависящих от дискретных переменных.

Благодарности. М. А. Манько благодарна организаторам Международной Конференции "Нелинейная физика. Теория и эксперимент. IV" (Галлиполи, Лечче, Италия, 2006) за гостеприимство и РФФИ за финансовую поддержку участия в конференции (грант № 06-02-26768).

\section{Список литературы}

[1] D. F. Styer, M. S. Balkin, K. M. Becker et al., Amer. J. Phys., 70 (2002), 288.

[2] S. Mancini, V. I. Man'ko, P. Tombesi, Phys. Lett. A, 213 (1996), 1.

[3] S. Mancini, V. I. Man'ko, P. Tombesi, Found. Phys., 27 (1997), 801.

[4] C. E. Shannon, Bell System Tech. J., 27 (1948), 379.

[5] O. V. Man'ko, V. I. Man'ko, J. Russ. Laser Res., 18 (1997), 407.

[6] M. A. Man'ko, J. Russ. Laser Res., 22 (2001), 168.

[7] O. V. Man'ko, V.I. Man'ko, J. Russ. Laser Res., 25 (2004), 115; quant-ph/0401131.

[8] O. V. Man'ko, V. I. Man'ko, G. Marmo, Phys. Scripta, 62 (2000), 446.

[9] O. V. Man'ko, V. I. Man'ko, G. Marmo, J. Phys. A, 35 (2002), 699.

[10] O.V. Man'ko, V.I. Man'ko, G. Marmo, "Tomographic map within the framework of star-product quantization", Quantum Theory and Symmetries, Proc. II Int. Symp. (Kraków, Poland, 2001), eds. E. Kapuschik, A. Horzela, World Sci. Publ., River Edge, NJ, 2002, 126.

[11] M. A. Man'ko, V.I. Man'ko, R. V. Mendes, J. Russ. Laser Res., 27 (2006), 507; quant-ph/0602189.

[12] A. Rènyi, Probability Theory, North-Holland Ser. Appl. Math. Mech., 10, North-Holland, Amsterdam, 1970.

[13] В. В. Додонов, В.И. Манько, Инварианты и эволюиия нестационарных квантовых систем, Тр. ФИАН, 183, Наука, М., 1987.

[14] I. Bialynicki-Birula, Phys. Rev. A, 74 (2006), 052101; quant-ph/0608116.

[15] S. De Nicola, R. Fedele, M. A. Man'ko, V. I. Man'ko, Eur. Phys. J. B., 52 (2006), 191; quant-ph/0607200.

[16] I. I. Hirschman, Amer. J. Math., 79 (1957), 152.

[17] I. Bialynicki-Birula, J. Mycielski, Commun. Math. Phys., 44 (1975), 129.

[18] M. A. Man'ko, V. I. Man'ko, R. Vilela Mendes, J. Phys. A, 34 (2001), 8321.

[19] G. M. D'Ariano, S. Mancini, V. I. Man'ko, P. Tombesi, Quant. Semiclass. Opt., 8 (1996), 1017.

[20] D. T. Smithey, M. Beck, M. G. Raymer, A. Faridani, Phys. Rev. Lett., 70 (1993), 1244.

[21] M. A. Man'ko, V. I. Man'ko, S. De Nicola, R. Fedele, Acta Phys. Hung. B, 26:1-2 (2006), 71. 